\title{
Variable Optical Feedback Based Behavioral Model of a Self-Mixing Laser Sensor
}

\author{
Usman Haider, Usman Zabit, Senior Member, IEEE, and Olivier D. Bernal, Member, IEEE \\ DOI:10.1109/JSEN.2021.3077251
}

\begin{abstract}
In this paper, a unified behavioral model of laser feedback based self-mixing interferometry (SMI) is proposed which is able to accurately model the SMI sensor signals encountered under experimental variable optical feedback conditions. The model provides correct SMI signals whether feedback is varied in a continuous or discrete manner, while spanning all major feedback regimes (such as weak-, moderate, and strong-feedback regime) used for sensing applications. As a result, the proposed model allows the simulation of SMI signals in the presence of speckle which is of upmost importance to develop future efficient algorithms to reconstruct target displacements. The optical speckle usually occurs when the target of comparable surface roughness to the laser wavelength is moving as it induces variation of the optical feedback factor. The proposed model is shown to be able to address such cases and in particular to be able to reproduce very similar SMI signals to those acquired in the presence of speckle. It is thus anticipated that the proposed model would facilitate the design and testing of novel SMI algorithms and systems dedicated to the processing of variable optical feedback based SMI signals for metric sensing applications.
\end{abstract}

Index Terms_- Variable optical feedback, self-mixing, laser sensor, speckle, interferometer.

\section{INTRODUCTION}

$\mathbf{L}$ ASER self-mixing interferometry (SMI) [1]-[3] is being widely used in laboratory and industrial environments for displacement, velocity (of fluids and solid targets), distance and vibration sensing [4]-[8]. Furthermore, mechatronic metrology, profilometry as well as biomedical applications have also flourished [9]-[11].

Importantly, SMI under stable and constant optical feedback (OF) is mature, with such SMI sensors already built and working in real-time conditions [12]-[15]. However, bigger challenge in further penetration of SMI instruments is their comparatively degraded performance in case of variable $\mathrm{OF}$, such as would occur due to speckle (caused by irregular surface of target) or due to change in sensor-to-target distance. Behavior of SMI sensor under constant OF is well-understood [1], [2] and modelled [16]. However, to the best of authors' knowledge, no such model exists in case of variable OF

Usman Haider and Usman Zabit are with the Department of Electrical Engineering, National University of Sciences and Technology (NUST), Islamabad 44000, Pakistan. (e-mail: usman.zabit@seecs.nust.edu.pk).

Olivier D. Bernal is with LAAS-CNRS, CNRS, Universite de Toulouse, Institut National Polytechnique Toulouse, ENSEEIHT, 31400 Toulouse, France

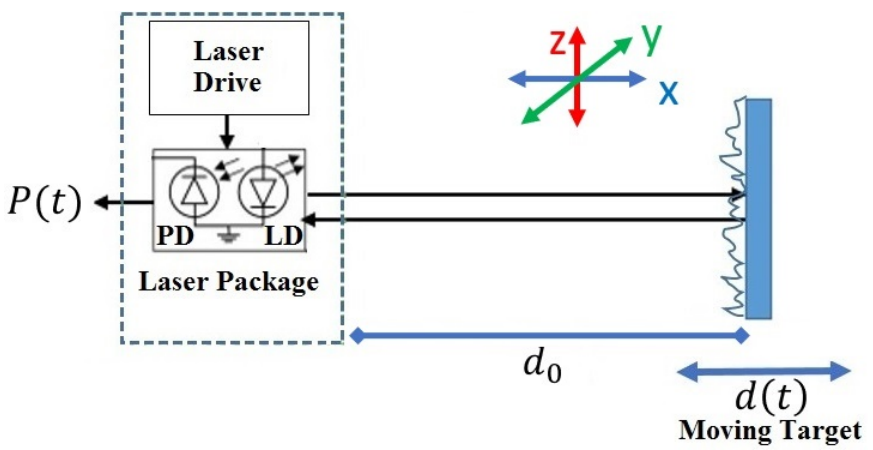

Fig. 1. Typical self-mixing laser sensor based on a laser-diode (LD) package with integrated photo-diode (PD), placed at a distance $d_{0}$ from remote target displacing with $d(t)$. Any motion component in the yz-plane results in variation of optical feedback due to rough target surface.

spanning all major OF regimes used for sensing applications.

In this paper, a unified behavioral model of SMI is proposed which is able to accurately model the SMI sensor signals encountered under experimental variable OF conditions. The model provides correct SMI signals whether OF is varied in a continuous or discrete manner, while spanning all major $\mathrm{OF}$ regimes. Furthermore, it matches the established model in case 
of constant OF [16]. It is envisioned that such a novel model would facilitate the design and testing of variable OF based SMI algorithms and systems for metric sensing applications.

The architecture of an SMI sensor is very simple and can be implemented using virtually any type of laser such as gas lasers, solid-state lasers, semiconductor lasers such as verticalcavity surface-emitting lasers (VCSELs) etc [1], [3], [17], [18]. However, semiconductor diode-laser is usually employed for SMI. Fig. 1] schematizes a typical SM sensor based on a laserdiode (LD) package inclusive of a photo-diode (PD) which is used to access the LD modulated power signal.

SMI sensing is also attractive because it can also work on a diffuser, rough surface or in presence of speckle [10], [17], [19]. Speckle effects in the SMI signal are simply observed as amplitude modulation of SMI signal, where fading of amplitude occurs repeatedly. Presence of speckle due to rough target can make signal processing difficult due to variable optical feedback. The fading of amplitude makes it difficult, if not impossible, to correctly detect the changes in the signal, resulting in significant erroneous measurements [17]. Effective operation of SMI technique on diffusing target surfaces and in the presence of speckle has been reported in multiple works, such as for measurement of displacement [20]-[23], velocity [24]-[26], and 3D profilometry [10]. Furthermore, given the complexity, development of new signal processing methods for SMI signals under variable optical feedback remains an active area of research.

The paper is structured as follows. Theory and fundamental equations of SMI are introduced in Section II. Section III discusses the behavioral model under constant feedback. Section IV presents our proposed behavioral model of a SM sensor with variable optical feedback. Results and conclusion will be presented in Section V and Section VI, respectively.

\section{SMI THEORY AND OPERATION}

Theory of SMI is well-documented [1], [2], and is briefly summarized below. The emitted laser reflects back from a remote surface and it interferes with the light that already exists inside the laser cavity. The laser threshold condition changes depending on the phase of feedback light. This results in change of emitted power for a given laser diode bias current. The optical power emitted by the LD subjected to optical feedback, or the so-called SMI signal, can be written as:

$$
P(t)=P_{o}\left\{1+m \cos \left[x_{F}(t)\right]\right\}
$$

where $P(t)$ is the power emitted by the LD with feedback, $P_{o}$ is the emitted power without feedback, $m$ is the modulation index, and $x_{F}(t)$ is the laser output phase in the presence of feedback. The shape of function $x_{F}(t)$ and value of modulation index $m$ depend on the optical feedback coupling parameter, $C$. The modulation index is given by [1]:

$$
m=\frac{2 \tau_{p}}{\tau_{c}} \epsilon \sqrt{R_{e x t}} \frac{1-R_{2}}{\sqrt{R_{2}}}=C \frac{\tau_{p} c}{L \sqrt{1+\alpha^{2}}}=C \cdot \gamma
$$

where $C$ is given by:

$$
C=\epsilon \sqrt{R_{e x t}} \frac{1-R_{2}}{\sqrt{R_{2}}} \frac{L \sqrt{1+\alpha^{2}}}{l . n}
$$

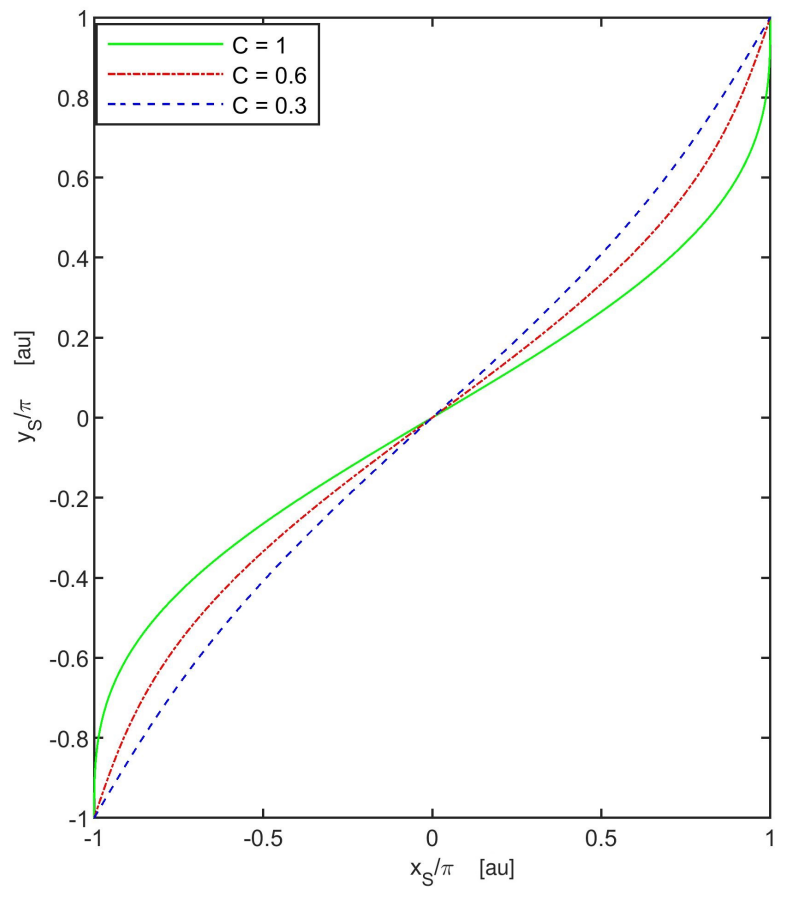

Fig. 2. Plot of $y_{S} / \pi$ versus $x_{S} / \pi$ for $\alpha=5$, satisfying the relation $x_{S}=y_{S}+C \sin \left(y_{S}\right)$. Solid green line represents $C=1$, red dash-dot line represents $C=0.6$ and blue dash-dash line represents $C=0.3$.

The coefficient $\epsilon$ is introduced to account for possible mode mismatch between the reflected light and the lasing mode, $\tau_{p}$ is the photon lifetime within the cavity, $\tau_{c}$ is the round trip time light takes within the interval cavity, $l$ is the laser cavity length, $n$ is the cavity's refractive index, $\alpha$ is the LD linewidth enhancement factor whose value is typically between 3 to 8 [27], $R_{\text {ext }}$ is the reflectivity of external target and $R_{2}$ is the reflectivity of laser mirror from which the light is emitted, while $L$ is the distance between the laser and the remote target.

If $d_{0}$ represents the initial distance between the laser and the target, and $d(t)$ represents the target's displacement along laser-axis, then $x_{F}(t)$ is given as:

$$
x_{F}(t)=2 \pi \frac{d(t)}{\frac{\lambda_{F}(t)}{2}}
$$

where $\lambda_{F}$ is the wavelength in presence of feedback. The output phase in the absence of feedback is given as:

$$
x_{0}(t)=2 \pi \frac{d(t)}{\frac{\lambda_{0}}{2}}
$$

where $\lambda_{o}$ is the wavelength in absence of feedback. Relation between $x_{F}(t)$ and $x_{o}(t)$ is given by the following excess phase equation [1]:

$$
x_{0}(t)=x_{F}(t)+C \sin \left[x_{F}(t)+\arctan (\alpha)\right]
$$

$C$ parameter plays a fundamental role in SMI as it determines the operating regime of the LD [1]:

1) For $0.1<C<1$, the regime is called weak feedback regime. This regime is described by weak optical 


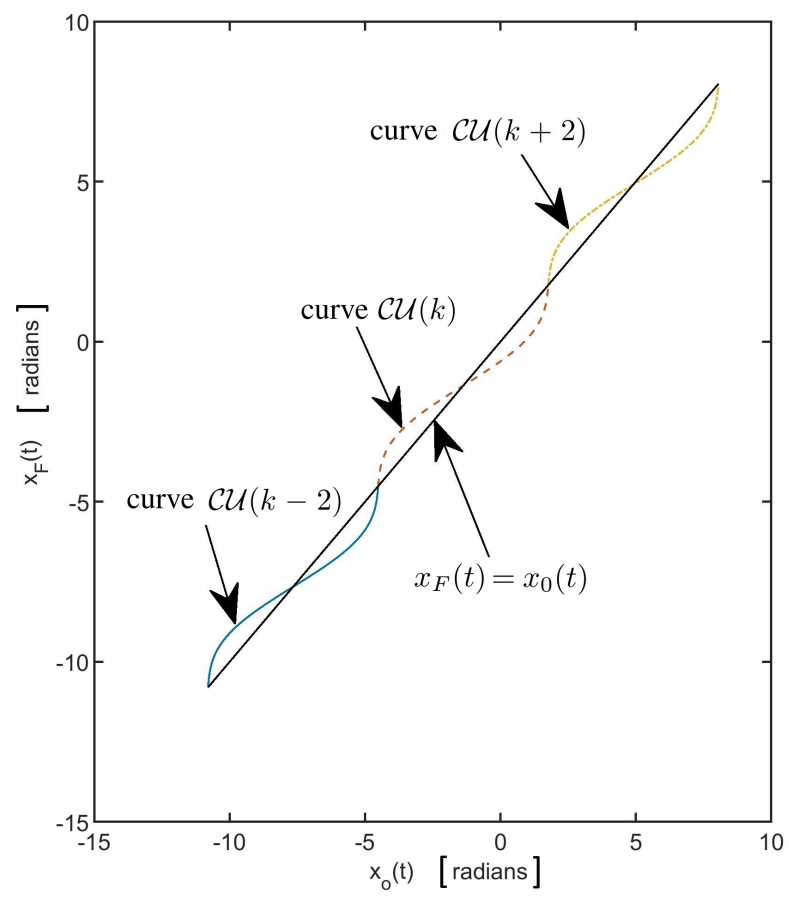

Fig. 3. Plot of $x_{F}(t)$ versus $x_{0}(t)$ for $C=0.9$ as juxtaposition of curves $\mathcal{C U}(k-2), \mathcal{C U}(k), \mathcal{C U}(k+2)$ and so on, with $k$ even.

feedback with only a single emission frequency and a broadening or narrowing of the emission line based on the phase of the feedback.

2) For $1<C<4.6$, we have the moderate feedback regime. The waveform looks like a sawtooth and shows hysteresis. The LD is no longer single mode as its phase condition becomes three-valued at some values of phase.

3) For $C>4.6$, the phase condition of the LD has more than three solutions. Experimentation becomes difficult because of the unstable spectral behavior of the LD.

\section{Behavioral Model for Constant Feedback}

In [16], a high-level model was presented to represent SMI phenomenon under constant OF. The model is able to compute the input/output relationship between the motion/displacement of a target and the laser output. The approach allowed the use of standard and powerful simulation tools such as MATLAB/Simulink to design new algorithms for signal processing of OF interferometers [28]-[30]. However, the work reported in [16] did not discuss SMI under variable optical feedback.

Specifically, authors of [16] divided the operating regimes of SM LD sensor in two regimes: either the value of feedback parameter satisfies $C<1$ or it is $C>1$. Their model is based on (6) that can also be expressed as follows:

$$
x=y+C \sin (y)
$$

where $x=x_{0}(t)+\arctan (\alpha)$ and $y=x_{F}(t)+\arctan (\alpha)$. The above relation can be represented in function-form as $x=$ $g(y ; C)$ where $g(y ; C)=y+C \sin (y)$.

\section{A. Model for $C<1$}

As stated in [16], for $C<1$, it can be seen that the samples $x_{S}$ can be easily computed from equally spaced samples $y_{S}$ in the range $-\pi$ to $\pi$. This makes it possible to plot $y_{S}$ versus $x_{S}$. This is shown in Fig. 2 for different values of $C$. A new function $y=f(x ; C)=g^{-1}(x ; C)$ where $g$ is the inverse of function $f$, defining a curve $\mathcal{C U}$, can be introduced and its direct calculation can be obtained using linear interpolation of samples from the data set $\left\{x_{S}, y_{S}\right\}$. This can be written as:

$$
y=f(x ; C) \approx f_{I N T}\left(x ; x_{S}, y_{S}, C\right)
$$

It is seen from Fig. 3 that, as mentioned in [16], the $x_{F}(t)$ plot can be obtained by a juxtaposition of the translated curves $\mathcal{C U}(k)$ of $\mathcal{C U}$ corresponding to $y_{S}$ in the range $(k-1) \pi$ to $(k+$ $1) \pi$ with $k$ even. Note that $\mathcal{C U}$ thus corresponds to $\mathcal{C U}(k=0)$, and depends on the value of $x_{0}(t)$ (which depends on the target displacement itself).

Value of $k$ is determined by,

$$
k(\text { even })=2 \times \operatorname{round}\left\{\frac{x_{o}(t)+\arctan (\alpha)}{2 \pi}\right\}
$$

where round $\{\ldots\}$ denotes rounding to the nearest integer.

Thanks to this juxtaposition method, it is thus only required to define the curve $\mathcal{C U}$ using the range of samples $y_{S}$ from $-\pi$ to $\pi$. Equation 8 is used to find the feedback phase, $x_{F}(t)$, from the displacement. The value of $k$ depends on the displacement and is given by (9).

\section{B. Model for $C>1$}

Similar to the case for $C<1,(8)$ is used to find the feedback phase, $x_{F}(t)$, from the displacement. However, due to the well-known hysteresis phenomenon affecting the feedback phase $x_{F}(t)$ for $C>1$ [1], the range of samples $y_{S}$ is from $-\beta$ to $\beta$, where $\beta$ is given by:

$$
\beta=\arccos \left\{-\frac{1}{C}\right\}
$$

The value of $k$ is determined by using infinite bank of triggers. Let us use $x_{0, R}(k)$ and $x_{0, F}(k)$ to denote the values of $x_{0}(t)$ at which there is a rising discontinuity and a falling discontinuity in $x_{F}(t)$, respectively. The functionality of trigger is then such that if $x_{0}(t)$ is greater than $x_{0, R}(k)$ the output of trigger is set to $\mathrm{ON}$ and it remains $\mathrm{ON}$ till $x_{0}(t)$ drops below $x_{0, F}(k)$. This trigger block functionality can be defined by using the relation [16]:

$$
q(t)=\left\{\begin{array}{cc}
1, & x_{0}(t) \geq x_{0, R}(k) \\
0, & x_{0}(t) \leq x_{0, F}(k) \\
\text { Hold, } & x_{0, F}(k)<x_{0}(t)<x_{0, R}(k)
\end{array}\right.
$$

where $x_{0, R}(k)$ and $x_{0, F}(k)$ are defined as [16]:

$$
\begin{array}{r}
x_{0, R}(k)=k \pi-\arctan (\alpha)+\beta+C \sin (\beta) \\
x_{0, F}(k)=(k+2) \pi-\arctan (\alpha)-\beta-C \sin (\beta)
\end{array}
$$




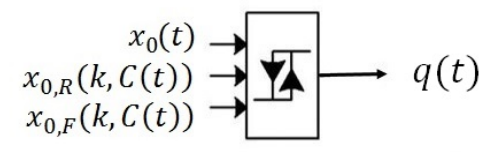

Fig. 4. Trigger block functionality under variable optical feedback.

\section{Proposed Variable Optical Feedback based BEHAVIORAL MODEL}

As stated earlier, variable OF frequently occurs during realworld SMI operation, especially in the presence of speckle due to rough target surface. The excess phase equation in case of variable optical feedback can be derived from (7) resulting in:

$$
x=y+C(t) \sin (y)
$$

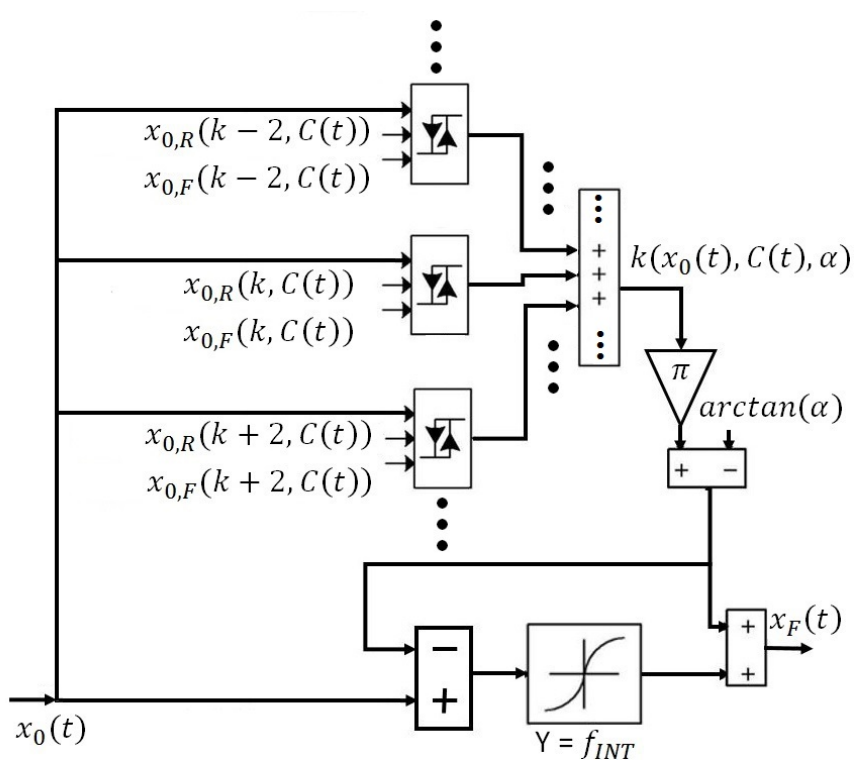

Fig. 5. Proposed unified behavioral model under variable optical feedback for both $C \leq 1$ and $C>1$.

Consequently, this implies that at every time-instant, the range of samples $y_{S}$ is changing, i.e $\beta(t)$ is changing. Thus, juxtaposition of similar translated curves $\mathcal{C U}(k)$ cannot be used in case of continuously varying $C(t)$ as the curve corresponding to each time-instant will be different. Thus, in the proposed model, a new curve is computed at each timeinstant in order to model instantaneous variation in $C(t)$.

In the variable OF model, samples $x_{S}$ can again be easily computed from equally spaced samples $y_{S}$. The range, $\beta(t)$, of the samples $y_{S}$ at every instant can be determined by using (10) when $C>1$ and by noting that in the absence of hysteresis for $C \leq 1, \beta(t)=\arccos \{-1\}=\pi$. Thus, we get the following function:

$$
\beta(t)=\left\{\begin{array}{cc}
\arccos \left\{-\frac{1}{C(t)}\right\}, & C>1 \\
\arccos \{-1\}, & C \leq 1
\end{array}\right.
$$

The calculation of $y=f(x ; C(t))$ from the data set $\left\{x_{S}, y_{S}\right\}$ can be done using interpolation as done earlier under constant feedback case. Phases $x_{0, R}(k)$ and $x_{0, F}(k)$ are now both dependent on instantaneous value of $C(t)$, given by:

$$
x_{0, R}(k, t)=k \pi-\arctan (\alpha)+\beta(t)+C(t) \sin (\beta(t))
$$

$x_{0, F}(k, t)=(k+2) \pi-\arctan (\alpha)-\beta(t)-C(t) \sin (\beta(t))$

In the case of constant feedback, $x_{0, R}(k)$ and $x_{0, F}(k)$ were dependent only on the value of $k$ which means dependence on $x_{0}(t)$. However, in this case of variable optical feedback, both of these phases are dependent on $k$ and $C(t)$ as well.

In this case, the trigger block depends on the instantaneous values of $\beta(t)$, which itself depends on the instantaneous value of variable optical feedback factor $C(t)$. If $x_{0}(t)$ is greater than or equal to $x_{0, R}(k, C(t))$ the trigger block is $\mathrm{ON}$ or the output is one, if it is less than or equal to $x_{0, F}(k, C(t))$, the trigger block is OFF or the output is zero, and if $x_{0}(t)$ satisfies $x_{0, F}(k, C(t)) \leq x_{0}(t) \leq x_{0, R}(k, C(t))$ condition then the trigger block holds its previous state. The output of new proposed trigger block can be defined by the relation:

$$
q(t)=\left\{\begin{aligned}
1, & x_{0}(t) & \geq x_{0, R}(k, C(t)) \\
0, & x_{0}(t) & \leq x_{0, F}(k, C(t)) \\
\text { Hold }, & x_{0, F}(k, C(t)) & <x_{0}(t)<x_{0, R}(k, C(t))
\end{aligned}\right.
$$

This new functionality of the trigger block is schematically presented in Fig. 4.

Importantly, by using the above-mentioned concept, one can unify the models presented earlier for $C<1$ and $C>1$. The modified model is based on the trigger block approach defined earlier with $\beta$ defined by (15). The new behavioral model for SMI LD with variable optical feedback is shown in Fig. 5

This unified model can be used for both $C \leq 1$ and $C>1$ as opposed to previous model which proposed two different models for SMI LD, one for $C<1$ and other for $C>1$. Also, this new model works equally well for variable optical feedback as well as constant optical feedback. Corresponding SMI signals generated using this model are presented next.

\section{RESULTS}

\section{A. Comparison with previous constant $C(t)$ model}

To validate the correct functionality of the proposed model, SMI signals are generated for different constant values of $C(t)$. SMI waveform based on the model in [16] are also generated for the sake of comparison. Open-access software GNU Octave has been used to implement these methods. Fig. 6 presents the SMI signal in weak feedback regime with $C=0.3$ under harmonic displacement of $5 \lambda_{0} / 2$. Likewise, moderate-regime constant $\mathrm{OF}$ case is also simulated for $C=3.2$ (see Fig. 7). It can be seen that the output of both the models are very similar. In fact, error between the two signals is zero in both cases. Thus, the proposed model can be used to model traditional SMI signals under constant $\mathrm{OF}$ as well, in addition to variable OF based SMI signals, as presented next.

Execution-time comparisons of proposed variable $C(t)$ model and previous constant $C$ model [16] were carried out for weak- and moderate-regime modelling for a signal with 

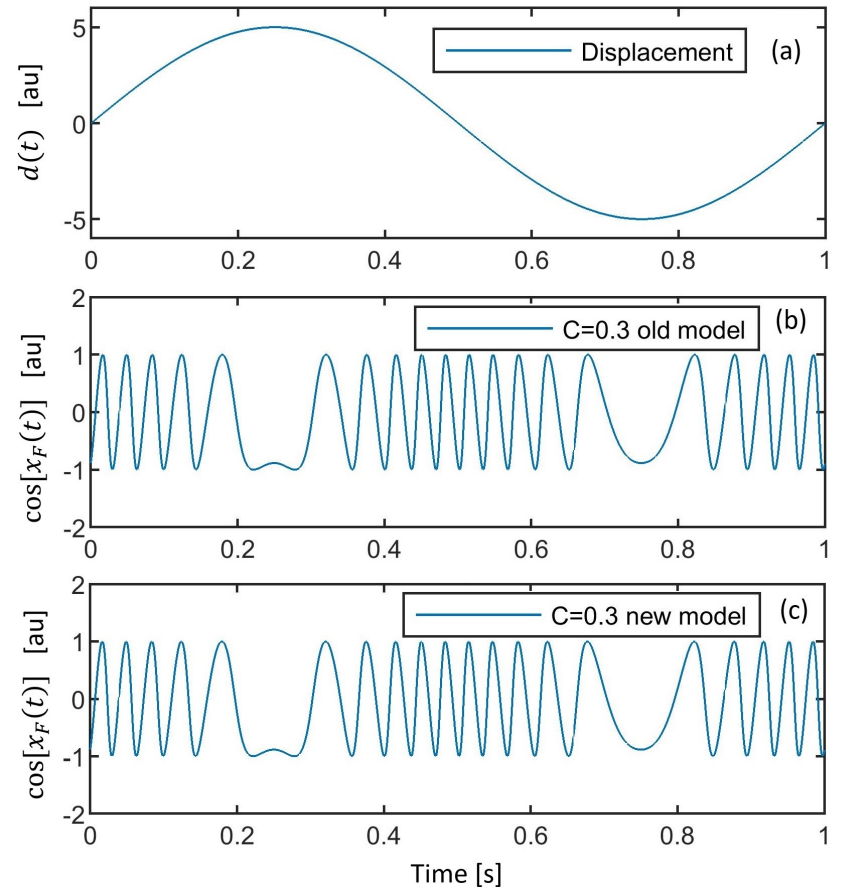

Fig. 6. Simulated SMI signal for $C=0.3$ and $\alpha=5$. (a) Target displacement $d(t)=5 \frac{\lambda_{0}}{2} \sin [2 \pi t]$, (b) old model's output [16], and (c) proposed model's output.

TABLE I. Execution-time comparisons of proposed variable $C(t)$ model and previous constant $C$ model [16] for weak- and moderate-regime modelling for a signal with $10^{4}$ samples.

\begin{tabular}{|c|c|c|c|c|}
\hline & \multicolumn{2}{|c|}{ Proposed model } & \multicolumn{2}{c|}{ Previous model [16] } \\
\hline & Weak-FB & Moderate-FB & Weak-FB & Moderate-FB \\
\hline & Time (s) & Time (s) & Time (ms) & Time (s) \\
\hline mean & 16.615 & 16.627 & 7.034 & 1.139 \\
\hline std & 0.154 & 0.069 & 0.431 & 0.009 \\
\hline
\end{tabular}

$10^{4}$ samples. Table I reports the mean and standard deviation (denoted as std) values after 10 repetitions on an Intel core-i7 computer with 8 GB RAM by using GNU Octave software. As expected, the proposed model is far more compute-intensive than the constant $C$ model [16] because it takes into account $C$ and $\beta$ variation for each time-sample. It is also interesting to observe the large difference in execution-time of the constant $C$ model [16] for weak- and moderate-FB cases because it uses different models for both regimes.

\section{B. Simulations under variable $C(t)$}

To simulate the variable optical feedback, a time varying $C$ signal is generated. This implies that as previously mentioned, the modulation index $m$ which can be estimated using (2), is also modified accordingly. Here, the value of $C$ is varied to increase linearly from 0.05 till 8 . It thus starts from very weak feedback regime and then increases so that it covers weak-, moderate- and strong-feedback regimes (see Fig. 87. As a result, assuming that $\alpha$ remains almost constant when $C$ is varying (see Fig. 5 in [31]), the resulting optical output power can then be approximated using (1). The target vibration frequency is set to $5 \mathrm{~Hz}$ with peak-to-peak displacement of $5 \lambda_{0}$. Fig. 8 presents the harmonic target motion, $C(t)$, and
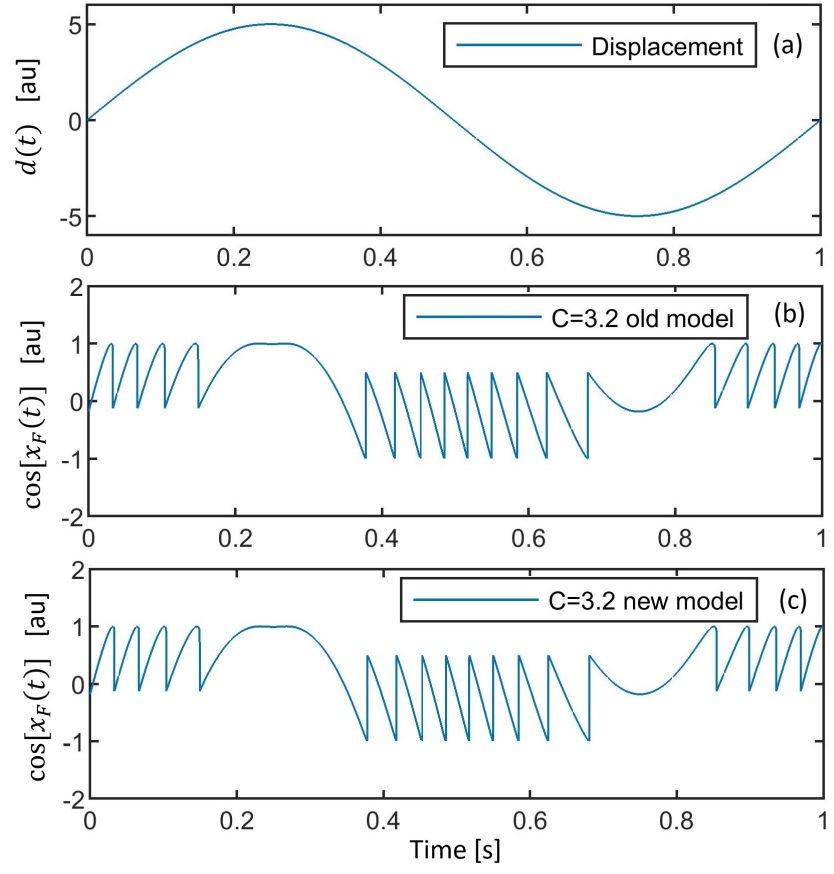

Fig. 7. Simulated SMI signal for $C=3.2$ and $\alpha=5$. (a) $d(t)=$ $5 \frac{\lambda_{0}}{2} \sin [2 \pi t]$, (b) old model's output [16], and (c) proposed model's output.

the SMI signal generated by the proposed model for this case. It can be seen that the shape and the amplitude of the generated SMI signal is directly dependent on the value of $C(t)$. This kind of behavior is consistent with observations of experimental SMI signals corresponding to variable optical feedback. Fringe-loss phenomenon, occurring under strongfeedback regime, is also observed in the generated SMI signal [7]. (Fringe-loss occurs for large $C$ values because of decrease in the number of stable solutions of the excess phase equation (6) for a given phase stimulus due to increase in $C$ [7].) At time $0.3 \mathrm{~s}$, the signal contains ten fringes that correspond to displacement of $5 \lambda_{0}$. But for larger values of $C(t)>4.6$, fringe-loss occurs, e.g., at time $0.8 \mathrm{~s}$ there are nine fringes and at time $0.9 \mathrm{~s}$ there are only eight fringes remaining in the SM signal. This is consistent with the theory of fringe-loss under strong optical-feedback regime for such large values of $C(t)$ [7].

Fig. 9 shows the SMI signal when the variations in $C(t)$ occur in a discrete manner. Here, $C(t) \in\{0.25,1.3,2.4,0.6\}$. The objective is to present the observation that the proposed model does not fail under such a case. The target vibration frequency is $5 \mathrm{~Hz}$ with peak-to-peak displacement of $5 \lambda_{0}$. The output clearly indicates the effect of instantaneous value of $C(t)$ on the amplitude and shape of the fringes, as described in (2). The shape of fringes is cosine like when the instantaneous value of $C(t)$ is $<1$. The fringes become sawtooth-shaped for the values of $C(t)>1$. Thus, the proposed model is also able to withstand discrete changes in $C(t)$. In fact, any type of remote motion $d(t)$ can be modelled because the proposed method computes the output sample of $P(t)$ for each samplepair of input data-vectors corresponding to $d(t)$ and $C(t)$. 

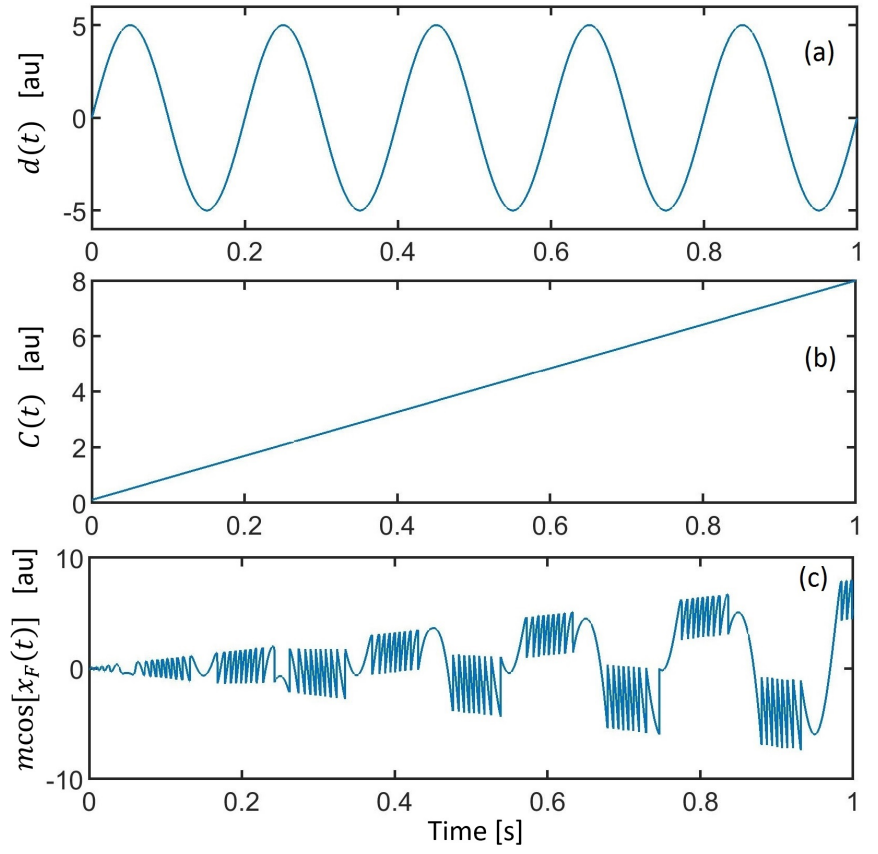

Fig. 8. Simulated SMI signal corresponding to linearly variable optical feedback under harmonic target motion of $5 \mathrm{~Hz}$ with amplitude of $5 \lambda_{0} / 2$ shown in (a). (b) Linearly variable optical feedback $C(t)$ spanning very weak-, weak-, moderate- and strong-optical feedback regimes, and (c) generated SMI signal as per proposed model.

\section{Modelling of experimental SMI signals with variable $C(t)$}

In order to validate our proposed model and to establish that the proposed model can be used to model practical experimental SMI signals with variable optical feedback, different such experimental SMI signals $P_{\text {exp }}(t)$ are used. The signals were acquired by using an SMI sensor based on a HL7851 laser diode with emission wavelength of $785 \mathrm{~nm}$ and $50 \mathrm{~mW}$ output optical power. No temperature stabilization system was used for the laser-diode as it remained stable under optical feedback. Built-in photo-diode located at the back-facet of laser diode package was used to acquire the sensor signal. The laser-diode package was placed inside the collimation tube (LT110P-B by Thor Labs) having a focusing lens of focal length $6.24 \mathrm{~mm}$. The collimated laser beam was directed at the remote target. A commercial piezoelectric transducer (PZT) from Physik Instrumente (P753.2CD) was deployed as remote target. Note that the SM sensor was displaced in the yz-plane (see Fig. 1) as well during operation (as would occur during embedded sensing applications [32]) to cause the laser beam to scan the PZT's metallic surface, thereby causing significant variation in optical feedback.

Fig. 10 (a) presents such an experimental SMI sensor signal under variable optical feedback due to yz-plane sensor movement and harmonic target motion with frequency $f_{\text {tar }}=60$ $\mathrm{Hz}$ and amplitude $A_{\text {tar }}=50 \mu \mathrm{m}$ approximately. This signal is lowpass-filtered and normalized (denoted as $P_{N_{e x p}}(t)$ ) for ease of later comparison with corresponding simulated signal.

An estimation of variable feedback coupling factor $C_{e s t}(t)$ for this experimental SMI signal is required to model equivalent SMI signal. Different $C$ estimation methods have been
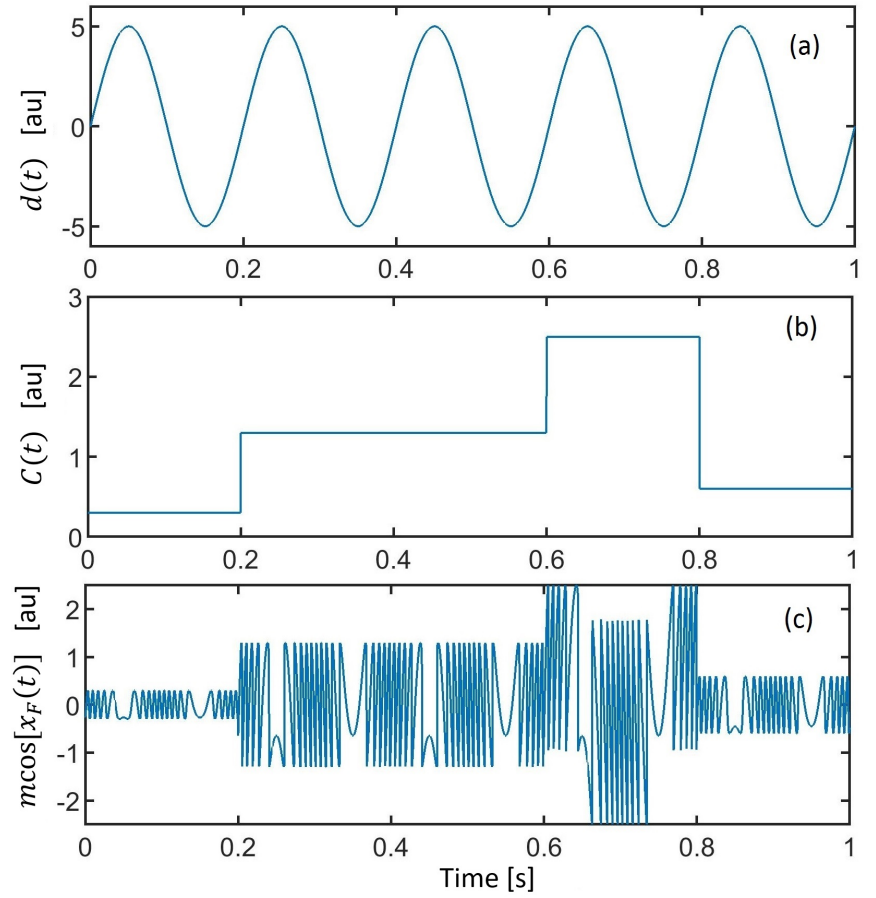

Fig. 9. Simulated SMI signal corresponding to discretely variable optical feedback under harmonic target motion of $5 \mathrm{~Hz}$ with amplitude of $5 \lambda_{0} / 2$ shown in (a). (b) Discretely variable optical feedback $C(t)$, and (c) generated SMI signal as per proposed model.

proposed in the literature [31], [33], [34]. However, for a given SMI signal acquisition, these algorithms provide an average estimation of $C$ and are not able to estimate timevarying $C$ corresponding to a given SMI signal. So, in this work, $C$ was individually estimated at certain time-instants (denoted by red vertical lines in Fig. 10 (c)) while $C_{\text {est }}(t)$ was obtained by using cubic interpolation [5] between these estimated $C$ values. Local $C$ estimation was performed at the start and the end of signal as well as for time-instants corresponding to local minima and maxima in $C(t)$ by using manual observation of the SM signal. This results here in nonuniform sampling (NUS) of $C(t)$ which is not a problem as NUS provides valid results so long as generalized ShannonNyquist criterion is satisfied [5]. (Note that $C_{e s t}(t)$ here spans the weak- and moderate-feedback regime, where weak optical feedback regime occurs due to speckle induced SMI sensor signal fading, as highlighted in Fig. 11.)

Then, by applying this $C_{e s t}(t)$ in the proposed model, corresponding simulated SMI signal is generated whose normalized version $P_{N_{\text {sim }}}(t)$ is plotted in Fig. 10 (d). (Other inputs to the proposed model included $x_{0}(t)=4 \pi \frac{d(t)}{\lambda_{0}}$, with $d(t)=A_{\text {tar }} \sin \left[2 \pi f_{\text {tar }} t\right], \lambda_{0}=785 \mathrm{~nm}$, and $\alpha=5$.) It can be seen by comparing Fig. 10 (b) and (d) that the proposed model is able to generate a signal very similar to the experimentally acquired variable optical feedback based SMI signal (see also the comparison of enlarged views in Fig. 11). It may also be elaborated that the objective here is neither an accurate measurement of $d(t)$ nor of $C(t)$ but to demonstrate that the proposed scheme is able to successfully model real-world variable feedback based SMI signals. Thus, slight deviations between the experimental SM signal and the modelled SM 

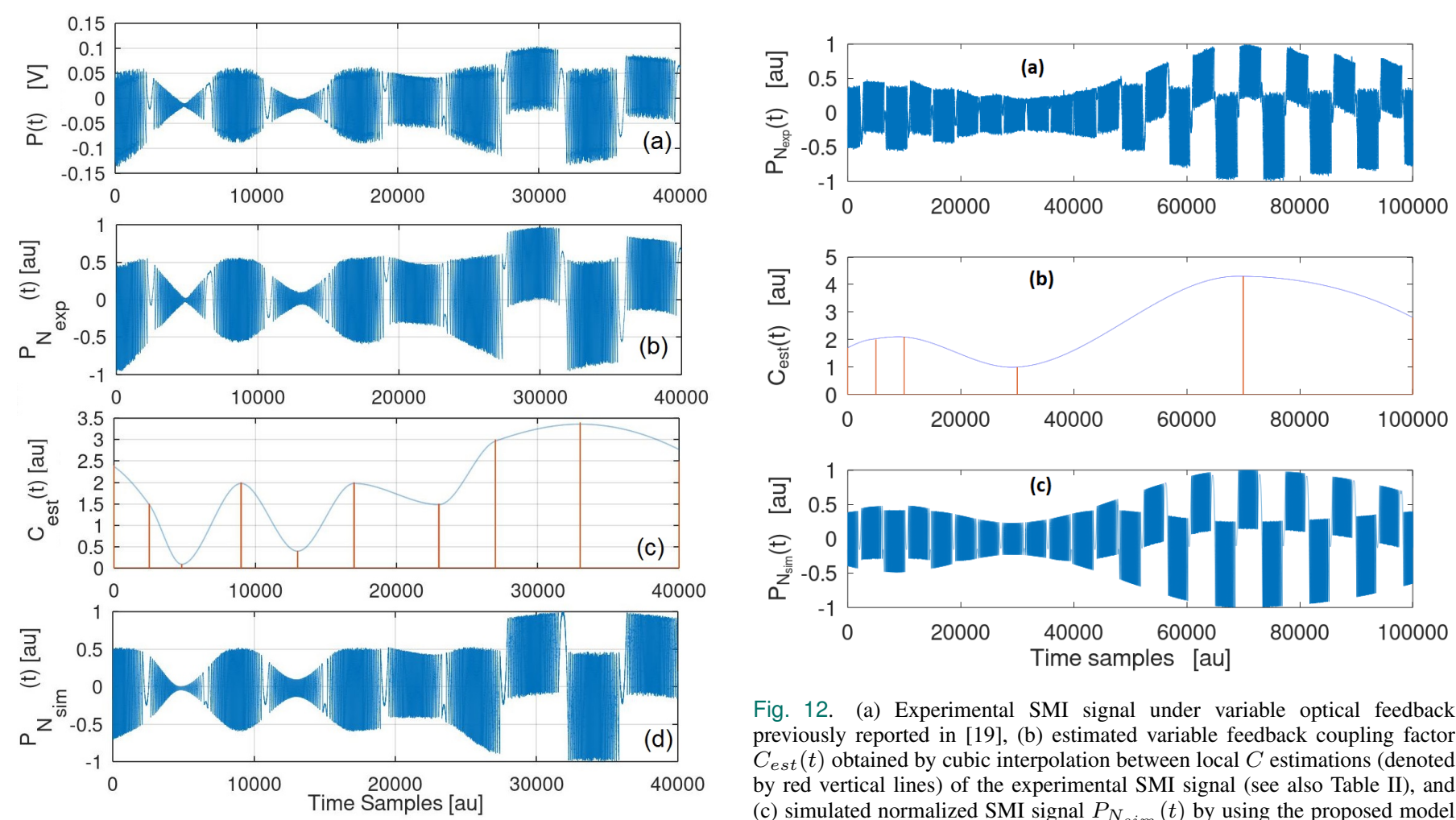

Fig. 12. (a) Experimental SMI signal under variable optical feedback previously reported in [19], (b) estimated variable feedback coupling factor $C_{e s t}(t)$ obtained by cubic interpolation between local $C$ estimations (denoted by red vertical lines) of the experimental SMI signal (see also Table II), and (c) simulated normalized SMI signal $P_{N_{s i m}}(t)$ by using the proposed model with $C_{\text {est }}(t)$.

Fig. 10. (a) Experimental SMI signal $P(t)$ under variable optical feedback and harmonic target motion, (b) filtered and normalized experimental signal $P_{N_{e x p}}(t)$, (c) estimated variable feedback coupling factor $C_{e s t}(t)$ based on local $C$ estimations (denoted by red vertical lines) of the experimental SMI signal followed by cubic interpolation between these local estimated $C$ values, and (d) simulated normalized SMI signal $P_{N_{s i m}}(t)$ by using the proposed model with $C_{\text {est }}(t)$ resulting in a signal similar to the experimental SMI signal.
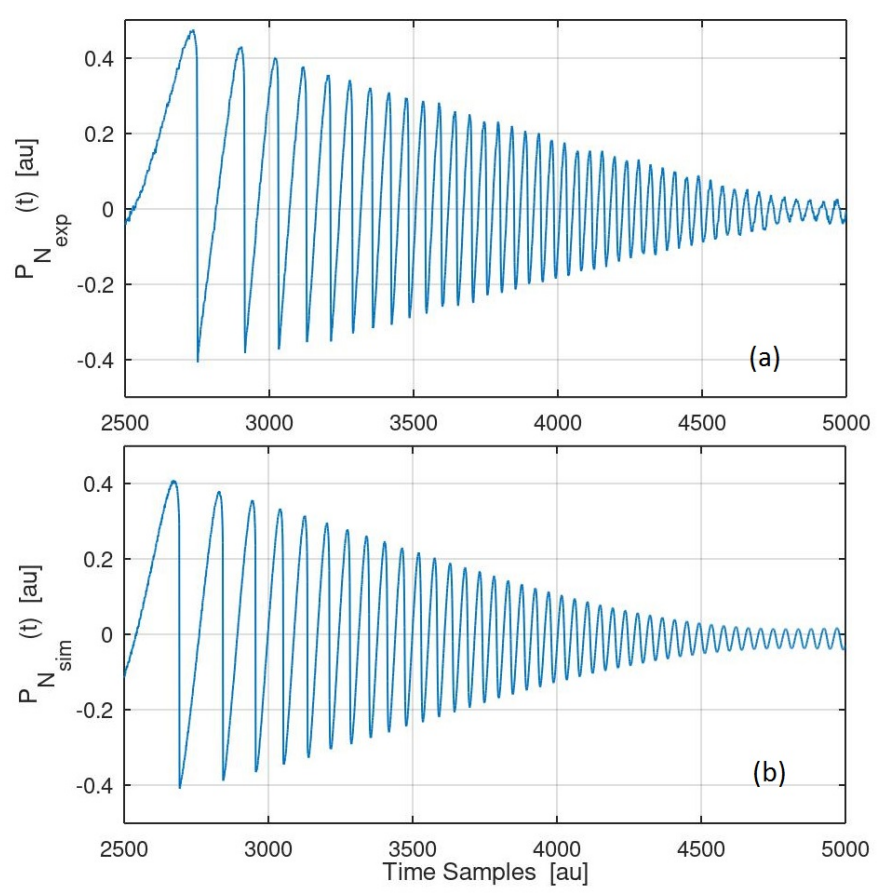

TABLE II. Local $C$ estimation values and corresponding time-samples for the experimental SMI signal under variable optical feedback previously reported in [19] and plotted in Fig. 12 (a).

\begin{tabular}{|c|c|c|c|c|c|c|}
\hline$C$ estimation & 1.7 & 2 & 2.1 & 0.99 & 4.3 & 2.8 \\
\hline Time-sample & 1 & $5 \times 10^{3}$ & $10^{4}$ & $3 \times 10^{4}$ & $7 \times 10^{4}$ & $10^{5}$ \\
\hline
\end{tabular}

signal will invariably exist due to imperfect knowledge of experimental $d(t)$ as well as of $C(t)$.

As another example, another variable optical feedback based SMI signal previously reported by authors in [19], is also modeled. The SMI signal occurred for target frequency of 60 $\mathrm{Hz}$ with $25 \mu \mathrm{m}$ peak to peak amplitude. Both the modeled SMI signal and the experimental SMI signal reported in [19] are shown in Fig. 12. Fig. 12 (b) presents the estimated optical feedback coupling signal $C_{\text {est }}(t)$, used to model the experimental signal. Table II presents the local $C$ estimation values used in cubic interpolation resulting in $C_{e s t}(t)$ and corresponding time-instants. It can be seen that the experimental SMI signal reported in [19] and the one simulated using the proposed model again closely match, thereby validating the proposed model.

\section{CONCLUSION}

A new high-level behavioral model is presented that is able to correctly model laser feedback based SMI sensors operating under variable optical feedback. The validity of the proposed model is checked by modelling the experimental SMI signals occurring under variable optical feedback, including those

Fig. 11. Enlarged view of a segment of : (a) experimental normalized signal $P_{N_{e x p}}(t)$ shown in Fig. 10 (b), and (b) simulated normalized SMI signal $P_{N_{\text {sim }}}(t)$ shown in Fig. 10 (d) by using the proposed model. reported earlier in literature. The new unified model is simple yet accurate and can be used to model SMI systems operating under variable feedback and works for any practical value of 
feedback, thus covering all major optical feedback regimes used for sensing applications inclusive of weak-, moderate, and strong-feedback regime. The proposed method extends the behavioral modeling capability of currently established, but constant optical feedback based method [16], by building upon its foundations. Thus, the proposed work exhibits similar strengths (simple modeling framework which can be easily integrated into existing simulation tools) and weaknesses (lack of modeling of transient-, or chaotic-behavior observed at very high laser feedback) as those of [16]. This novel model is envisioned to support the design and testing of SMI sensing algorithms and systems aiming to achieve high-accuracy measurements even under variable optical feedback conditions.

\section{REFERENCES}

[1] T. Taimre, M. Nikolić, K. Bertling, Y. L. Lim, T. Bosch, and A. D. Rakić, "Laser feedback interferometry: a tutorial on the self-mixing effect for coherent sensing," Adv. Opt. Photon., vol. 7, pp. 570-631, Sep 2015.

[2] S. Donati, "Developing self-mixing interferometry for instrumentation and measurements," Laser \& Photonics Reviews, vol. 6, no. 3, pp. 393 417, 2012.

[3] A. Valavanis, P. Dean, Y. L. Lim, R. Alhathlool, M. Nikolic, R. Kliese, S. P. Khanna, D. Indjin, S. J. Wilson, A. D. Rakic, et al., "Self-mixing interferometry with terahertz quantum cascade lasers," IEEE Sensors Journal, vol. 13, no. 1, pp. 37-43, 2012.

[4] L. Lu, W. Zhang, B. Yang, J. Zhou, H. Gui, and B. Yu, "Dual-channel self-mixing vibration measurement system in a linear cavity fiber laser," IEEE Sensors Journal, vol. 13, no. 11, pp. 4387-4392, 2013.

[5] O. D. Bernal, U. Zabit, F. Jayat, and T. Bosch, "Sub- $\lambda / 2$ displacement sensor with nanometric precision based on optical feedback interferometry used as a non-uniform event-based sampling system," IEEE Sensors Journal, vol. 20, no. 10, pp. 5195-5203, 2020.

[6] M. Norgia, A. Pesatori, and L. Rovati, "Self-mixing laser doppler spectra of extracorporeal blood flow: a theoretical and experimental study," IEEE Sensors Journal, vol. 12, no. 3, pp. 552-557, 2011.

[7] M. Veng, J. Perchoux, and F. Bony, "Fringe disappearance in self-mixing interferometry laser sensors: Model and application to the absolute distance measurement scheme," IEEE Sensors Journal, vol. 19, no. 14, pp. 5521-5528, 2019.

[8] S. Amin, U. Zabit, O. D. Bernal, and T. Hussain, "High resolution laser self-mixing displacement sensor under large variation in optical feedback and speckle," IEEE Sensors Journal, vol. 20, no. 16, pp. 9140-9147, 2020.

[9] S. Ottonelli, M. Dabbicco, F. De Lucia, M. Di Vietro, and G. Scamarcio, "Laser-self-mixing interferometry for mechatronics applications," Sensors, vol. 9, no. 5, pp. 3527-3548, 2009.

[10] S. Donati and G. Martini, "3d profilometry with a self-mixing interferometer: Analysis of the speckle error," IEEE Photonics Technology Letters, vol. 31, no. 7, pp. 545-548, 2019.

[11] J. Perchoux, A. Quotb, R. Atashkhooei, F. J. Azcona, E. E. RamírezMiquet, O. Bernal, A. Jha, A. Luna-Arriaga, C. Yanez, J. Caum, et al., "Current developments on optical feedback interferometry as an alloptical sensor for biomedical applications," Sensors, vol. 16, no. 5, p. 694, 2016.

[12] A. Ehtesham, U. Zabit, O. D. Bernal, G. Raja, and T. Bosch, "Analysis and implementation of a direct phase unwrapping method for displacement measurement using self-mixing interferometry," IEEE Sensors Journal, vol. 17, pp. 7425-7432, Nov 2017.

[13] G. Giuliani, S. Bozzi-Pietra, and S. Donati, "Self-mixing laser diode vibrometer," Measurement Science and Technology, vol. 14, no. 1, p. 24, 2002.

[14] A. Magnani, A. Pesatori, and M. Norgia, "Self-mixing vibrometer with real-time digital signal elaboration," Applied Optics, vol. 51, no. 21, pp. 5318-5325, 2012

[15] T. Hussain, S. Amin, U. Zabit, O. D. Bernal, and T. Bosch, "A high performance real-time interferometry sensor system architecture," Microprocessors and Microsystems, vol. 64, pp. 23 - 33, 2019.

[16] G. Plantier, C. Bes, and T. Bosch, "Behavioral model of a self-mixing laser diode sensor," IEEE Journal of Quantum Electronics, vol. 41, pp. 1157-1167, Sep. 2005.
[17] L. Lu, K. Zhang, J. Dai, J. Zhu, S. Zhen, and B. Yu, "Study of speckle pattern effect for self-mixing laser diodes in vertical-cavity surfaceemitting lasers," Optical Engineering, vol. 49, no. 9, p. 094301, 2010.

[18] Y. Zhao, J. Zhou, C. Wang, Y. Chen, and L. Lu, "Temperature measurement of the laser cavity based on multi-longitudinal mode laser selfmixing effect," IEEE Sensors Journal, vol. 19, no. 12, pp. 4386-4392, 2019.

[19] U. Zabit, O. D. Bernal, and T. Bosch, "Self-mixing laser sensor for large displacements: Signal recovery in the presence of speckle," IEEE Sensors Journal, vol. 13, pp. 824-831, Feb 2013.

[20] A. A. Siddiqui, U. Zabit, O. D. Bernal, G. Raja, and T. Bosch, "All analog processing of speckle affected self-mixing interferometric signals," IEEE Sensors Journal, vol. 17, pp. 5892-5899, Sep. 2017.

[21] M. Norgia, S. Donati, and D. D'Alessandro, "Interferometric measurements of displacement on a diffusing target by a speckle tracking technique," IEEE Journal of Quantum Electronics, vol. 37, pp. 800806, June 2001.

[22] M. Norgia and S. Donati, "A displacement-measuring instrument utilizing self-mixing interferometry," IEEE Transactions on Instrumentation and Measurement, vol. 52, pp. 1765-1770, Dec 2003.

[23] O. D. Bernal, H. C. Seat, U. Zabit, F. Surre, and T. Bosch, "Robust detection of non-regular interferometric fringes from a self-mixing displacement sensor using bi-wavelet transform," IEEE Sensors Journal, vol. 16, no. 22, pp. 7903-7910, 2016.

[24] S. K. Ozdemir, S. Takamiya, S. Ito, S. Shinohara, and H. Yoshida, "Selfmixing laser speckle velocimeter for blood flow measurement," IEEE Transactions on Instrumentation and Measurement, vol. 49, pp. 10291035 , Oct 2000

[25] G. Plantier, N. Servagent, A. Sourice, and T. Bosch, "Real-time parametric estimation of velocity using optical feedback interferometry," IEEE Transactions on Instrumentation and Measurement, vol. 50, pp. 915919, Aug 2001.

[26] R. Atashkhooei, S. Royo, and F. J. Azcona, "Dealing with speckle effects in self-mixing interferometry measurements," IEEE Sensors Journal, vol. 13, pp. 1641-1647, May 2013.

[27] M. Osinski and J. Buus, "Linewidth broadening factor in semiconductor lasers-an overview," IEEE Journal of Quantum Electronics, vol. 23, no. 1, pp. 9-29, 1987.

[28] U. Zabit, O. D. Bernal, S. Amin, M. F. Qureshi, A. H. Khawaja, and T. Bosch, "Spectral processing of self-mixing interferometric signal phase for improved vibration sensing under weak- and moderatefeedback regime," IEEE Sensors Journal, vol. 19, pp. 11151-11158, Dec 2019.

[29] A. L. Arriaga, F. Bony, and T. Bosch, "Real-time algorithm for versatile displacement sensors based on self-mixing interferometry," IEEE Sensors Journal, vol. 16, no. 1, pp. 195-202, 2015.

[30] Z. A. Khan, U. Zabit, O. D. Bernal, and T. Hussain, "Adaptive estimation and reduction of noises affecting a self-mixing interferometric laser sensor," IEEE Sensors Journal, vol. 20, no. 17, pp. 9806-9815, 2020.

[31] Y. Yu, G. Giuliani, and S. Donati, "Measurement of the linewidth enhancement factor of semiconductor lasers based on the optical feedback self-mixing effect," IEEE Photonics Technology Letters, vol. 16, no. 4, pp. 990-992, 2004.

[32] U. Zabit, O. D. Bernal, and T. Bosch, "Design and analysis of an embedded accelerometer coupled self-mixing laser displacement sensor," IEEE Sensors Journal, vol. 13, no. 6, pp. 2200-2207, 2013.

[33] C. Bes, G. Plantier, and T. Bosch, "Displacement measurements using a self-mixing laser diode under moderate feedback," IEEE Transactions on Instrumentation and Measurement, vol. 55, no. 4, pp. 1101-1105, 2006.

[34] Y. Gao, Y. Yu, J. Xi, and Q. Guo, "Simultaneous measurement of vibration and parameters of a semiconductor laser using self-mixing interferometry," Applied Optics, vol. 53, no. 19, pp. 4256-4263, 2014. 